Rainer Bayreuther (Freiburg i. Br.)

\title{
Friedrich Kittlers Bedeutung für die Musikwissenschaft
}

\section{Disziplinarität}

Der Medienwissenschaftler Friedrich Kittler ist am 18. Oktober 2011 gestorben. Sein Tod ist kein guter Anlass, seine Bedeutung oder vielmehr seine Herausforderung an die Musikforschung in den Blick zu nehmen. Man hätte es schon früher tun können. Von seinen ersten Schriften an spielt die akustische Dimension von Medien eine zentrale Rolle. Allerdings hat er sich in den letzten Jahren mit dem ersten Band seiner auf vier Bände angelegten Studie Musik und Mathematik (2006 und 2009) ausdrücklich der Musik zugewandt. Muss die Musikwissenschaft die kühnen Behauptungen eines Medienwissenschaftlers ernst nehmen? Soll sie sich wirklich mit Sätzen wie „Vor Liebe summen uns die Ohren“ oder „Die Welt des Hörens und der Ohren geht Pythagoras also auf wie sonst nur Sappho ihre Liebe“1 befassen, die wie aus dem disziplinären Jenseits gesprochen anmuten und an den gegenwärtigen Diskurs im Fach beim besten Willen nicht anschlussfähig erscheinen?

Ja, sie sollte. Sie ist gut beraten, Kittlers Überlegungen zu Musik und Musikgeschichte zu bedenken. Ich habe die Vermutung, dass Kittlers Denken der Musik einige blinde Flecken der disziplinären Musikforschung aufdeckt. Political Correctness verabscheute nicht nur Kittler, sie wäre auch in der Auseinandersetzung mit ihm fehl am Platz. Es geht schlicht um plausible oder unplausible Argumente.

Kittler provoziert mit dem, was er sagt, und damit, wie er es sagt. Eine weitere Provokation besteht darin, dass es sich ein Vertreter der Medienwissenschaft herausnimmt, als musikwissenschaftlicher Dilettant Grundlegendes zu Musik und Musikgeschichte zu sagen. Das ruft pauschale Abwehrreflexe hervor. Auch von der Zunft, aus der er ursprünglich stammt, in der er promoviert wurde und sich habilitierte, der germanistischen Literaturwissenschaft, wird Kittler mit derartigen Reflexen abgewehrt. Es scheint nicht unnötig zu sagen, dass man mit etwaigen sachlichen Fehlern in Kittlers Denken der Musik nach den Grundsätzen guter wissenschaftlicher Praxis umgehen sollte. Erstens sollte geprüft werden, ob eine als falsch vermutete Behauptung falsifizierbar ist, um ihr zweitens gegebenenfalls eine besser begründete Behauptung entgegenzustellen. Drittens wäre zu überlegen, ob Kittlers strittige Behauptung die Plausibilität seines Ansatzes insgesamt tangiert; wenn nein, ist es damit getan, den Fehler richtigzustellen, wenn ja, ist allgemeinere Kritik erlaubt und geboten. Schließlich sollte man sich nicht deshalb dazu hinreißen lassen, diese Regeln des Argumentierens zu brechen, weil sich Kittler selbst nicht an sie gehalten hat.

\section{Einschreiben}

Wie viele Medienwissenschaftler spezifiziert Kittler das Prädikat „schreiben“ durch mehrere Präfixe: „aufschreiben“, „anschreiben“, „einschreiben“. Wenn man etwa davon spricht, dass ein Komponist ein Musikstück schreibt, dann meinen die Spezifizierungen Folgendes: Der

1 Friedrich Kittler: Musik und Mathematik I/1: Aphrodite, München 2006, S. 236. 
Komponist schreibt das Stück auf, das heißt, er bringt es in irgendeiner musikalischen Notation zu Papier. Der Komponist schreibt die Klänge des Stücks a n, das heißt, er bringt bestimmte Parameter des Stücks (Töne, Akkorde, Dauern usw.) in eine bestimmte symbolische Form. Der Komponist schreibt sich mit dem Auf- und Anschreiben in ein Medium ein, das heißt, er nimmt die Handlungsoptionen und gegebenenfalls auch die Bedeutungsoptionen, die das Medium bietet, in Anspruch. Letzteres ist für die Medienwissenschaft der springende Punkt, denn an diesem Punkt situiert das berühmte medienwissenschaftliche Theorem des Medienaprioris: „The medium is the message“. Man darf sich nicht davon irritieren lassen, dass Kittler gegen Marshall McLuhan, den Urheber des Theorems, gern polemisiert hat. Kittlers Denken der Musik läuft just darauf hinaus, dass es unsachgemäß ist, zu sagen, Musik habe/repräsentiere/symbolisiere/transportiere eine „message“. Sie ist selbst ihre „message“. Damit ist ganz und gar nicht gesagt, dass Musik immer abstrakt bliebe, immer L'art pour l'art sei. Kittler verfolgt das fundamentalontologische Programm Martin Heideggers, das Verb „ist“ des Satzes „Musik ist selbst ihre message“ auszulegen. Dazu muss er analysieren, worin sich musikalisches Agieren einschreibt.

Soll sich die Musikwissenschaft dafür interessieren? Ja, sie sollte, denn die Behauptung, es sei unsachgemäß zu sagen, Musik habe/repräsentiere/symbolisiere/transportiere eine „message“, beinhaltet fundamentale Weichenstellungen des musikalischen Verstehens.

Mit dem Begriff „Aufschreibesysteme“ im Titel seiner literaturwissenschaftlichen Habilitationsschrift wurde Kittler schlagartig berühmt. ${ }^{2}$ Die Herausforderung für die Literaturwissenschaft lag darin, dass hier nicht Literatur als solche Gegenstand der Untersuchung war, sondern eine Reihe von Medien, in die ein konkretes Stück Literatur sich einschreibt und die zu einem Teiltod des Autors beitragen, indem ihnen ein gutes Stück der eigentlichen Autorschaft zukommt. Einen besonders kontrovers diskutierten Grund entlehnt Kittler von Jacques Lacan ${ }^{3}$ und Roman Jakobson ${ }^{4}$ : Jeder neu geborene Mensch hat seine absolut individuellen Laute, bis ihm die Mutter eine vorbegriffliche Ur-Sprache einflüstert. Diese Ur-Sprache wird dann zum Medium, in die der heranwachsende Mensch und schließlich der reife Dichter sein Sprechen und Dichten einschreibt. Dieses im frühen Lebensalter erworbene Lautsystem ist die Basis dafür, dass die Dichter uns ein Bedeutungsnetz von aufeinander bezogenen, kausal und sinnhaft verbundenen Sachverhalten, kurzum: eine Welt er-dichten können. Weil dieses Lautsystem der begrifflichen Welt vorgelagert ist, ist es auch relevant für die nichtbegriffliche musikalische Kunst, denn ebenso er-komponieren die Komponisten musikalische Welten, das heißt Netze von musikalischen Sachverhalten, die als Klang- und Rhythmussysteme oder als akustische Zeitsysteme an der Welt partizipieren. Das Medium ist hier in mehrerer Hinsicht Referenz: Bedeutungsreferenz, Wahrhaftigkeitsreferenz, Referenz weiterer sprachlicher Pragmatiken, ${ }^{5}$ Referenz der Klanglichkeit. Die Plausibilität der jeweils er-dichteten oder er-komponierten Welt wird vom Medium approbiert.

2 Friedrich Kittler, Aufschreibesysteme 1800/1900, München ${ }^{1} 1985,{ }^{2} 1987,{ }^{3} 1995,{ }^{4} 2003$. Die zweite Auflage ist geringfügig, die dritte und vierte jeweils stark überarbeitet.

3 So bereits in Aufschreibesysteme 1800/1900, in extenso ausgeführt in Friedrich Kittler, Dichter Mutter Kind, München 1991.

4 Friedrich Kittler, Musik und Mathematik I/2: Eros, München 2009, S. 93.

5 Den Aspekt der sprachlichen Pragmatik, der alle nicht-bedeutungshaften Aspekte der Sprache bezeichnet, entlehne ich von Herbert Paul Grice, Studies in the Way of Words, Cambridge/MA 1989. Wahrhaftigkeit (Glaubwürdigkeit) einer Aussage sei im Folgenden als eine Implikatur der Aussage im Sinn von Grices Pragmatik aufgefasst. 
Soll sich die Musikwissenschaft dafür interessieren? Ja, sie sollte, ohne dass sie sich verpflichtet fühlen muss, Kittlers psychoanalytische Herleitung zu akzeptieren. Denn in Kittlers These, dass es ein klangliches, aber vorbegriffliches Medium des Dichtens gibt, ist eine These über die Relation von Musik und Dichtung enthalten. Das eine kann nicht der Ursprung des anderen sein. Weder „prima la musica e poi le parole“ noch umgekehrt. Vielmehr wachsen beide auf dem Boden eines vorausliegenden Systems. Die These ist falsifizierbar; ihre beiden Falsifikate lauten: Zuerst, und zwar entwicklungspsychologisch, paläoanthropologisch, neurologisch, kompositionsgeschichtlich usw. zuerst, die Sprache, dann die Musik bzw. umgekehrt. Man müsste weit ausholen, um das Für und Wider der Alternativen zu diskutieren. Aber es kann kein Zweifel bestehen, dass eine solche Diskussion jede Fallanalyse des Verhältnisses von Musik und Sprache flankieren müsste, angefangen vom Wort-Ton-Verhältnis in der mittelalterlichen Einstimmigkeit durch die Jahrhunderte bis zur Verwendung von Sprache als kompositorischem Material im 20. und 21. Jahrhundert. Die Interpretation jedes Pink-Floyd-Songs, jeder Strauss-Oper, jedes Taktes von Wagners Götterdämmerung, ja selbst eines wortlosen Bach-Präludiums in der Interpretation von Glenn Gould - um als beliebige Beispiele einige Hausgötter Kittlers zu nennen - ist herausgefordert von Kittlers Behauptung, dass hier nicht einfach nach Gutdünken des Komponisten Worte und Töne zueinanderfinden, sondern dass das Zueinander von einer vorgängigen Ordnung durchherrscht ist, die durch eine gewöhnliche Interpretation der Musik vom Text her nicht erreicht werden kann. Und es steht seine herausfordernde Behauptung im Raum, dass diese Ordnung an Stellen an die ästhetische Oberfläche drängt, die von den etablierten Methoden der musikalischen Analyse schlecht erfasst werden, zum Beispiel in den allerersten Tönen eines Stückes.

An der psychoanalytischen Deutung hängt die Plausibilität von Kittlers medientheoretischem Ansatz in der Tat nicht. Aber sie hängt an dem, worauf Lacan hinauswollte: dass die vorgängige Ordnung, in die sich Komponiertes und Gesagtes einschreiben, nichts mit dem seelischen Innenleben oder mit der Selbstreflexivität eines idealistisch aufgefassten Subjekts zu tun hat. Vielmehr ist es ein Sich-erstrecken des musikalischen oder sprachlichen Akteurs in eine vorgängige Ordnung, eine Beziehung zum Beispiel zu seiner Mutter, zu seinem Vater, zu Dingen und Menschen, ein ek-sistere, das als Ek-sistenz die Welt dieses Akteurs bildet. Die Ordnung, in die sich Kunstwerke einschreiben, ist ontischer - und nicht psychischer, nicht anthropologischer, auch nicht kultureller - Natur. Der medientheoretische Gedanke des Einschreibens wird damit zu einem höchst bedenkenswerten Ansatz, um zu erklären, wie es zu politischen, religiösen, sozialen usw. Charakteristiken von Musik kommt. Indem sich ein Akteur in eine mediale Ordnung erstreckt, spannt sich eine Welt auf. Je nach der Art und Weise des Erstreckens ist dies dann eine politische, eine religiöse, eine familiäre usw. Welt. In diese Welt geht die mediale Ordnung als eine - wie ich unten ausführen werde - Bedingung der Wahrhaftigkeit von Aussagen ein. (So möchte ich das Theorem „The medium is the message“ präzisieren.) Aber auch die Musik wirkt an der Ausgestaltung der Welt mit, indem sie sich in die Weise des Sich-erstreckens einmischt. Genau dadurch bildet sie die Charakteristik der Welt mit. Das ist eine weit plausiblere Beschreibung diesseits der unbefriedigenden Auffassung, Musik sei irgendein ästhetisches Ding sui generis und werde von psychischen, politischen, religiösen, sozialen usw. Gegebenheiten bloß semantisiert. 
Die Ordnung, in die sich musikalische und andere Kunstwerke einschreiben, ist beim frühen Kittler der Diskurs (Foucault) ${ }^{6}$, beim späteren Kittler das Medium (McLuhan). ${ }^{7}$ Beim frühen Kittler dominiert der Diskurs das Medium. Beim späteren Kittler dominiert das Medium den Diskurs, der aber als Theorieelement seine Bedeutung einbüßt. Das sind zwei unterschiedliche, ja konträre Auffassungen darüber, welche Ontologie vorgängige Ordnungen haben. Muss sich die Musikwissenschaft damit befassen? Ja, denn sie sollte eigentlich ein Wort mitzureden haben dabei, auf welche Weise uns eine vorgängige Ordnung zugänglich und wie sie materialiter beschaffen ist. Hier muss eine Unterscheidung getroffen werden, die bei Kittler nur allgemein als Übernahme von McLuhans zweitem medienwissenschaftlichem Theorem auftaucht, dass der Inhalt eines Mediums wiederum ein Medium sei. Ein konkretes Musikstück schreibt sich ein in vorgängige Ordnungen. Es kann aber auch selbst eine vorgängige Ordnung für nachgelagerte Äußerungen sein. Ein - sagen wir - Schubertlied ist nicht nur auf eine mediale Ordnung angewiesen, die über die Möglichkeiten von Sinnproduktion und Sinnverstehen geböte. Es ist auch selbst ein Medium, das regelt, welche Sinnmöglichkeiten und welche Pragmatiken der verbalen Schicht realisiert und wie sie übermittelt werden. Es ergibt sich dann die Frage, ob sich die beiden Ordnungen verschiedener Stufe wechselseitig bedingen und was sie miteinander zu tun haben. Sind diese Ordnungen verschiedener Stufe im Foucault'schen Sinn diskursiv? Oder müssen sie ontologisch anders gefasst werden? In Kittlers Werk gibt es in dieser Frage einen Umbruch, ohne dass Kittler sich damit explizit auseinandergesetzt hätte.

Die Musik ist in die Frage geradezu existenziell involviert, und zwar auf zwei Ebenen: Erstens, indem sie selbst Teil der medialen Ordnung ist, in die sich Aussagen und Handlungen des politischen oder religiösen Daseins einschreiben. Der gesamte Typus der empirischen musiksoziologischen Studien, der danach fragt, wie sich Menschen in ihrem Denken, Fühlen und Handeln von Musik beeinflussen lassen, gehört hierher. Zweitens, indem sich jedes musikalische Agieren - gleich ob produktiv, nachschaffend oder rezeptiv - in mediale Ordnungen einschreibt, die im weitesten Sinn musisch, aber kein konkretes Musikstück sind: die erwähnten welthaltigen Klang-, Rhythmussysteme oder akustischen Zeitsysteme. Auf diese zweifache Weise erhält Musik ihre Charakteristik und wird zum Beispiel religiöse oder politische Musik. Sie prägt die jeweilige Daseinscharakteristik mit, indem sie erstens als Medium selbst einem bestimmten kulturellen Bereich ihre spezifische ästhetische Färbung verleiht, und indem sie zweitens als konkretes Musikstück oder als konkrete musikalische Handlung die Information ist, die auf einem vorgängigen Medium prozessiert wird. Indizien dafür gibt es in Fülle. In den zwei grundlegenden menschlichen Existenzweisen Religion und Politik hat man auf Schritt und Tritt mit Musik zu tun. Die Religion hat sich seit alters her bei der Musik für ihr Handeln bedient; die Politik agiert spätestens mit dem Siegeszug der technischen Repräsentationsmedien Radio und Fernsehen und seit Kurzem mit dem „totalen Medienverbund auf Digitalbasis“8 im Sound und als Sound.

Der medientheoretische Zugriff auf die Musik, so kann Kittlers Ansatz präzisiert werden, fördert also zwei elementare Sachverhalte zutage:

6 Urszenen. Literaturwissenschaft als Diskursanalyse und Diskurskritik, hrsg. von Friedrich Kittler und Horst Turk, Frankfurt a. M. 1977.

7 Siehe ausführlich und exzellent dargestellt bei Geoffrey Winthrop-Young, Friedrich Kittler zur Einführung, Hamburg 2005.

8 Friedrich Kittler, Grammophon Film Typewriter, Berlin 1986, S. 8. 
1. Die Welt hat musische Merkmale (neben anderen Merkmalen selbstverständlich). Daher hat sie die Kraft, Aussagen, Handlungen, Artefakte usw. als wahrhaftig, das heißt gültig vor dem Horizont der Welt, zu beglaubigen.

2. Der Umgang mit musikalischen Artefakten - produktiv, nachschaffend, rezeptiv - ist eine Weise, sein Dasein zu erstrecken, um in dem erstreckten Raum zu leben. Die Voraussetzung dafür, dass dies gelingt, ist, dass das musikalische Artefakt sich in die Welt der ersten Ebene auch einfügt.

Beide Ebenen haben also einen im weitesten Sinn musischen Aspekt. Daher ist jede Vereinseitigung der Rolle der Musik bei der Ausbildung von Daseinscharakteristiken falsch. Die fragliche Ontologie einer vorgängigen Ordnung ist also immer dann auch eine musikwissenschaftliche Fragestellung, wenn die Ordnung musische Merkmale hat (1.) bzw. wenn die auf ihr errichtete Daseinscharakteristik musikalische Aspekte aufweist (2.). Auch wenn Kittler die erforderliche Präzision der Ausführung vermissen lässt, auch wenn dasjenige, was der frühe Kittler Medium nennt und der späte nur noch erzählend umschreibt, einer ontologischen Analyse bedarf: Es bleibt sein Verdienst, die Musikforschung an diese ihre Aufgabe erinnert zu haben. Wenn sie sie vernachlässigt, dann früher oder später um den Preis ihrer gesellschaftlichen Relevanz.

\section{Totale Mobilmachung}

Wohl am meisten erregte Kittler die Gemüter mit seinen notorischen Querverweisen auf Krieg und Militär. Das beginnt mit dem Buch Grammophon Film Typewriter (1986), setzt sich in vielen teils ungedruckten Vorträgen fort und endet erst beim ersten Band von Musik und Mathematik (2006). Dort spart Kittler sein Lieblingsthema aus, wobei ich vermute, dass er es in den geplanten Bänden Hesperien und Turingzeit wieder aufgenommen hätte.

Was hat Medienwissenschaft mit Militär zu tun, und sollen wir uns allen Ernstes aufgefordert fühlen, eine Trias Medium/Musik/Militär zu denken?

Kittler ist der Auffassung, zwei zentrale moderne Entwicklungen seien ursächlich mit Militär und Krieg verbunden: Massenmedien und Digitalisierung. Die Kameratechnik des Fernsehens etwa sei aus der militärischen Aufklärungstechnik entstanden, der Kameragebrauch aus dem Schusswaffengebrauch, die Übertragungs- und Verschlüsselungstechniken aus der Kommunikation des Militärs. ${ }^{9}$ Dafür muss sich die Musikwissenschaft nicht interessieren. Dass die Rockmusik ein Abkömmling der Kriegstechnik ist, wie Kittler behauptet, ${ }^{10}$ fällt durchaus in ihr Aufgabengebiet. Solche direkten Kausalitäten kann man mit guten Gründen bestreiten. Um die Tragweite der Dinge zu erfassen, ist es jedoch angeraten, den Zusammenhang zwischen Musik und Militär an seiner medialen Basis zu erörtern, und das ist die Digitalisierung. In der Digitalisierung seit den 1980er Jahren gelangt, so glaubt Kittler, eine Entwicklung zu ihrer massentechnischen Umsetzung, die seit dem frühen 20. Jahrhundert anwesend ist. Ihr epochales Signum ist der Gedanke der allgemeinen Mobilmachung, wie er in den 1920er und 30er Jahren aufkam. Um diesen Zusammenhang zu erläutern, müssen wir etwas ausholen.

9 Friedrich Kittler, Optische Medien, Berlin 2002, S. 39, 290 und passim, in: Grammophon Film Typewriter, S. 352.

10 Friedrich Kittler, „Rockmusik - ein Missbrauch von Heeresgerät“, in: Short Cuts, Frankfurt a. M. 2002, S. 7-30. 
Das Wesen der Digitalisierung besteht darin, die symbolischen Codes aller beliebigen Medien - Verbalsprache, Buchstabenschrift, durmolltonale Musik, Notenschrift, Zahlen, mathematische Kalküle usw. - in abstrakte, nicht-symbolische Ein/Aus-Zustände umzuwandeln. Dadurch lassen sich alle Medien nach der Zeit korrelieren, nach beliebigen mathematischen Algorithmen kombinieren und letztlich ineinander überführen. Die digitale Schaltung ist das Supermedium aller Medien. Es ermöglicht eine nicht-symbolische Verflüssigung aller Gehalte und Semantiken, die vormals nur in symbolischen Medien repräsentiert werden konnten. Die digitale Welt ist notwendigerweise eine Welt, die die vielen Welten abgeschlossener Sinnzusammenhänge, Sprachräume, Kulturen und Zeichenpraktiken kassiert.

Genau das war in den 1920er Jahren das Wesen von Totalitarismus ${ }^{11}$ und totaler Mobilmachung. ${ }^{12}$ Im Stadium der totalen Mobilmachung werden alle geschlossenen Welten aufgelöst und in eine einzige überführt. Diese einzige Welt ist nolens volens eine politische Welt, denn in ihr kommen alle Menschen und alle kulturellen Praktiken zusammen. Sie ist aber auch, und darauf will Kittler hinaus, eine totale materialistische Elementarisierung. Im Stadium der totalen Mobilmachung ist es irrelevant, ob ein gegebener Klang eine Autohupe, ein Ausschnitt aus einer Beethoven-Symphonie oder die innere akustische Empfindung eines bestimmten Menschen zu einem bestimmten Zeitpunkt ist. Relevant ist einzig, dass die Ereignisse im Fall eines Falles austauschbar und beliebig operabel - und das heißt medientechnisch: digital - sind. Meines Erachtens braucht man die Kategorien Krieg und Militär nicht, um die Koinzidenz plausibel zu machen. Totalitarismus und totale Mobilmachung waren ursprünglich keine Beschreibungen militärischer oder kriegerischer Ereignisse. Heidegger, der den Begriff der totalen Mobilmachung in den 1930er Jahren kritisch analysiert hat, hat ihn als Wesen der modernen Technik insgesamt aufgefasst, nicht nur der Kriegstechnik. ${ }^{13}$ Dass die Kriege des 20. Jahrhunderts nach den Spielregeln der totalen Elementarisierung alles Gegebenen funktionierten, ist offenkundig, sie sind aber nicht die Ursache der totalen Elementarisierung.

Dies alles fällt in das Aufgabengebiet der Musikwissenschaft. Denn die Musik spätestens ab dem 19. Jahrhundert hat an dieser Entwicklung Anteil. Dreieinhalb Beispiele:

1.a) In der deutschen Musikwissenschaft der 1920er Jahre war das Musische ein Label dafür, dass Musik in der Lage ist, unterschiedlichste Lebensbereiche zu koppeln: Arbeit, Religion, Sport, Wehrdienst, Feste, Bräuche. In der Musikpädagogik Fritz Jödes oder Georg Götschs, in der phänomenologischen Musikforschung Heinrich Besselers, Wilibald Gurlitts, Walter Wioras oder Friedrich Blumes und in der politischen Ästhetik Carl Schmitts, Hans Freyers oder Ernst Kriecks war die Musik mehr als die anderen Künste ein Medium, das eine totale Übersetzbarkeit aller Lebensbereiche im Sinn einer totalen Mobilmachung ermöglichte.

1.b) An der historischen Schwelle zum Nationalsozialismus wurde erstmals in voller Schärfe die Möglichkeit der politischen Eskalation der Musik erfasst. Die Voraussetzung für diese Eskalation ist darin gegeben, dass das Politische, das bisher in den symbolischen

11 Zur Genese des Begriffs im faschistischen Italien siehe Jens Petersen, „Die Geschichte des Totalitarismusbegriffs in Italien“, in: ,Totalitarismus' und ,Politische Religionen: Konzepte des Diktaturvergleichs, hrsg. von Hans Maier, Paderborn u. a. 1996, S. 15-35.

12 Der Begriff wurde von Ernst Jünger, „Die totale Mobilmachung“, in: Krieg und Krieger, hrsg. von dems., Berlin 1930, ed. in: ders., Essays 1 (= Sämtliche Werke 7), Stuttgart 1980, S. 119-142, geprägt.

13 Martin Heidegger, Beiträge zur Philosophie (Vom Ereignis), Frankfurt a. M. 1989 (verfasst in den späten 1930er Jahren), Abschnitt 74. 
Gestalten der Institutionen, Stände, der Verfassung und ihrer Organe usw. eingeschlossen war, atomisiert wurde in elementare, und das heißt: nicht-symbolische, nicht-humane Vorgänge der Gemeinschaftsbildung. Erst in dieser Atomisierung, die medientheoretisch einer Digitalisierung entspricht, wird das politische Potenzial des Ästhetischen - gleichsam medienarchäologisch - freigelegt. Die Möglichkeit des Politischen von Musik muss meines Erachtens mit den medientheoretischen Kategorien gedacht werden, die in Kittlers Konnex von Digitalisierung und totaler Mobilmachung enthalten sind.

2. Bei Wagner wird ab Tristan und Isolde (1865), teilweise schon bei Vorläuferfiguren wie Chopin, Berlioz und Liszt, nicht mehr auf der symbolischen Ebene komponiert. Das heißt, die Komposition besteht nicht mehr aus musikalischen Gestalten, die als solche Gegenstand der Wahrnehmung und der Interpretation des Rezipienten sind. Wagner komponiert unter der Oberfläche des genuin Musikalischen. Er komponiert direkt auf der Klaviatur der Wahrnehmung und des Gefühls. Oder mit Friedrich Nietzsches Worten: Wagner komponiert nicht Musik, Wagner komponiert musikalische Wirkpotenziale. ${ }^{14}$ Das bedeutet - medientheoretisch gesagt - eine Digitalisierung der Musik. Musik wird in eine Medialität überführt, die an ausnahmslos alles anschlussfähig ist und alles aneinander anschließen kann. Kittler hat sich selbst immer wieder mit Wagner beschäftigt ${ }^{15}$ und aus dieser Diagnose, die der von ihm verachtete Theodor W. Adorno noch wagnerkritisch vortrug, ${ }^{16}$ eine radikale anthropologische Behauptung abgeleitet: Wagners Musik bringt an den Tag, dass der Mensch - und der moderne Mensch zumal - eine Wahrnehmungs- und Emotionsmaschine ist.

3. Die Atomisierung des musikalischen Materials in der Zwölftontechnik, und dann in einer zweiten Eskalationsstufe nach 1945, führte zu einer Auflösung der symbolischen Einheit der musikalischen Parameter. Die Parameter konnten nun auf absolut beliebige Weise miteinander korreliert und ineinander überführt werden. Die Eigenqualität der Parameter setzte dem keine Grenzen mehr. Nicht zufällig gingen serielle und elektronische Musik nach 1945 Hand in Hand. Der Einsatz der Elektronik war eine konsequente technische Umsetzung der Tatsache, dass die kompositorische Ebene in der seriellen Musik auf das akustische Supermedium schlechthin verlagert wurde, das die Einzelmedien der vormaligen musikalischen Parameter in ein und demselben Material modellieren konnte. Dieses Material ist die Sinusschwingung, die eine klangliche Qualität (Amplitude und Phasenwinkel) in die Zeitdimension (Periodendauer) erstreckt. Für den menschlichen Hörer bedeutet diese Digitalisierung eine Totalisierung. Sie verwischt komplett die Grenzen, anhand derer in der menschlichen Wahrnehmung akustische Kontinuen in distinkte Klangereignisse (Klang/Stille, hoch/tief, laut/leise, Einzelton/Akkord usw.) separiert werden. Dem wahrnehmenden Menschen entgleitet damit auch die Möglichkeit, zwischen distinkten Klangereignissen und anderen distinkten mentalen Einstellungen (Emotionen, beliebige

14 „Wagner rechnet nie als Musiker, von irgend einem Musiker-Gewissen aus: er will die Wirkung, er will Nichts als die Wirkung. Und er kennt das, worauf er zu wirken hat!“ Friedrich Nietzsche, „Der Fall Wagner“, in: ders., Sämtliche Werke, hrsg. von Giorgio Colli und Mazzino Montinari, München 1980, durchgesehene Aufl. 1988, Bd. 6, S. 9-53, hier: S. 31

15 Am ausführlichsten in Grammophon Film Typewriter. Vgl. auch die Vorträge „Das Nahen der Götter vorbereiten“ (2008) und „Weltatem. Über Wagners Medientechnologie“ (1984), erschienen in: Friedrich Kittler, Das Nahen der Götter vorbereiten, München 2011.

16 Theodor W. Adorno, „Versuch über Wagner“ (ursprünglich „Fragmente über Wagner") in: Zeitschrift für Sozialforschung 8 (1939/40), S. 1-48; erweitert unter dem endgültigen Titel erschienen München 1964, ed. in: ders., Die musikalischen Monographien, Frankfurt a. M. 1971 (= Gesammelte Schriften 13), S. 7-148. 
vorgestellte Semantiken, Körperwahrnehmungen usw.) stabile Relationen herzustellen. Der Mensch kommt in einen Zustand totaler rezeptiver und mentaler Mobilmachung. Das ist seit der seriellen Musik im Wesentlichen unsere musikalische Situation.

\section{Musik}

Kittler hat sich en passant schon immer mit Musik auseinandergesetzt. Erst mit seinen letzten Büchern aber rückte die Musik in den Mittelpunkt seiner Arbeit, nachdem in den 1980 er Jahren die verbalsprachlichen Medien ${ }^{17}$ und in den 1990er Jahren Computer, Militärisches und visuelle Medien ${ }^{18}$ im Zentrum gestanden hatten. Die Wendung hin zur Musik war zugleich eine Hinwendung zu den alten Griechen. Zusammen mit der 2010 jung verstorbenen Rechtswissenschaftlerin Cornelia Vismann schrieb Kittler das Buch Vom Griechenland (Berlin 2002). Das schmale Bändchen nimmt das Großprojekt Musik und Mathematik in nuce vorweg. Es schrieb Wissenschaftsgeschichte und reihte sich selbst in eine andere Wissenschaftsgeschichte ein. Es läutete die großen Forschungsprojekte zur Antike ein, die heute an den Berliner Universitäten angesiedelt sind. Kittler gefiel sich offenkundig aber auch darin, eine ältere wissenschaftsgeschichtliche Volte nachzuvollziehen, nämlich Heideggers Kehre der 1930er Jahre. So wie Heidegger sich von der Analyse der Existenz in der Moderne in der von ihm selbst so genannten Kehre den Vorsokratikern zugewandt hatte, so kehrte sich Kittler nun den Vorsokratikern zu, und zwar speziell deren Musik.

Zu Kittlers Rezeption der Philosophie Heideggers sei noch bemerkt: Obwohl Kittlers Denken den bislang umfänglichsten Versuch darstellt, Heidegger für die Musikforschung fruchtbar zu machen, legt Kittler die konkreten Bezüge zu Heidegger nie offen. Wenn er ihn erwähnt, dann in einer Mischung aus Bewunderung und Koketterie. Im einzigen Text, der explizit von Heidegger handelt, sagt er, er ziehe es vor, „über manche Denker nicht zu schreiben, sondern von ihnen her" ${ }^{\text {"19. }}$.

Kittler hat kein speziell musikhistorisches Erkenntnisinteresse. Musikhistorische Beiträge ergeben sich nebenbei und stehen unter dem breiteren Dach einer umfassenden Seinsgeschichte des Abendlandes. Diese Seinsgeschichte ist für Kittler eine Mediengeschichte. Die angemessene Methodik ist folglich eine medienarchäologische. In dieser Seins- bzw. Mediengeschichte muss, so behauptet Kittler, die Musik einen bevorzugten Platz einnehmen, aber nicht sie allein: Musik, Zahl und Alphabetschrift stehen gleichursprünglich und eng ineinander verflochten am Anfang der abendländischen Seinsgeschichte. Die visuellen Medien hingegen wie auch kulturelle Ausprägungen wie Religion und Metaphysik gehören späteren Stadien an.

Wie bei Heidegger ist bei Kittler die Seinsgeschichte eine Verfallsgeschichte. In dieser Verfallsgeschichte bekommt die Musikgeschichte eine geradezu exzeptionelle Rolle. Während Heidegger den Untergang des Abendlandes erst spät beginnen lässt, bei René Descartes im 17. Jahrhundert, ist für Kittler das musikalische Denken schon bei Platon, Aristo-

17 Neben der Habilitationsschrift Aufschreibesysteme 1800/1900, vor allem die Texte „Carlos als Carlsschüler", in: Goethes und Schillers Literaturpolitik, hrsg. von Wilfried Barner u. a., Stuttgart 1984, S. $241-$ 273; Grammophon Film Typewriter; Dichter Mutter Kind.

18 „Farben und/oder Maschinen denken“, in: Synthetische Welten. Kunst, Künstlichkeit und Kommunikationsmedien, hrsg. von Eckhard Hammel, Essen 1996, S. 119-132; Optische Medien; Short Cuts.

19 Friedrich Kittler, „Heidegger und die Medien- und Technikgeschichte. Oder: Heidegger voraus“, in: Heidegger-Handbuch, hrsg. von Dieter Thomä, Stuttgart 2003, S. 500-504. 
teles und Aristoxenos ein Symptom des Verfalls. Der gesamte zweite Teilband von Musik und Mathematik I zielt auf den Nachweis, dass bereits 400 v. Chr. das musikalische Denken der homerischen und pythagoreischen Zeit nicht mehr verstanden wurde. Von da aus lässt sich der mutmaßliche Skopus der geplanten weiteren Bände recht genau umreißen:

Im Band Musik und Mathematik II: Roma aeterna und seinen beiden Teilen „Sexus" und „Virginitas“ wollte sich Kittler mit der Musik der vorchristlichen (Sexus) und der christlichen (Virginitas) lateinischen Welt auseinandersetzen, wo sich das Missverstehen und Umdeuten der Musik der ganz alten Griechen weiter fortsetzt. ${ }^{20}$

Im Band Musik und Mathematik III: Hesperien weist die geographische Angabe Hesperien (der Westen) in Hölderlins Elegie Brod und Wein den Weg: Im westlichen und späten Teil des Abendlandes, dem Europa der Renaissance und der Neuzeit, haben die „entflohenen Götter“ eine „Spur“ hinterlassen, wie Hölderlin dichtet, und diese Spur wollte Kittler in den drei Teilen „Minne“ (Renaissance), „Liebe“ (18. und 19. Jahrhundert) und „Sex“ (Moderne) verfolgen. Diese Spur der alten Götter der homerischen Zeit und ihrer Musik wäre also in den Blütezeiten der kunstvollen Mehrstimmigkeit aufzuzeigen gewesen. Es wäre zu einem Aufeinandertreffen einer Fortschritts- und Aufstiegsgeschichte der europäischen Musikkultur, wie sie von der etablierten Musikgeschichtsschreibung gesehen wird, mit der verfallsgeschichtlichen Sichtweise des Seins- und Mediengeschichtlers gekommen. Ein gewisser Spektakelwert wäre zu erwarten gewesen.

Den abschließenden Band Musik und Mathematik IV: Turingzeit plante Kittler ohne Unterteile. Dahinter kann man Programm vermuten. Mit der von Alan Turing ausgedachten virtuellen Maschine wird nichts weniger als das Ende der Geschichte eingeläutet. Historische Stadien hat es seitdem und wird es auch künftig keine mehr geben, denn die Digitalisierung ist ein irreversibler Prozess. Mit der Turingmaschine ist der mathematisch strenge Beweis verbunden, dass alle Behauptungen genau dann mit Ja/Nein bzw. Wahr/ Falsch verifizierbar sind, wenn sie durch eine Turingmaschine modelliert werden können. Und diese Modellierung ist denkbar einfach, sie kommt mit den drei Operationen Schreiben, Lesen und Schreib-/Lesekopf bewegen aus und kann technisch einfach implementiert werden. Oder umgekehrt: Alle Fragen, die entscheidbar sind, können auch im digitalen Supermedium des Ein- und Auslesens eines einzigen Informationsgehalts (1 oder Nicht-1) auf ein beliebiges Speichermedium modelliert werden. Wenn man akzeptiert, dass eine historische Epoche als der Horizont von Gründen aufgefasst werden kann, aus denen letzten Endes alle Fragen mit Ja/Nein bzw. Wahr/Falsch beantwortet wurden, dann wäre mit der Turingzeit in der Tat das Ende der Geschichte erreicht. Die Turingzeit wird letzten Endes nur noch Fragen akzeptieren, die digital modellierbar sind, und alle anderen als sinnlos verwerfen. Es wird darin auch nur graduelle Perfektionierung, aber keine fundamentalen historischen Umbrüche mehr geben, denn die völlige Allgemeingültigkeit der Turingmaschine ist bewiesen. Und man muss nicht nur erwarten, sondern kann es an den Veränderungen der gegenwärtigen Universitätslandschaft allenthalben beobachten, dass sich die digitale Modellierung sukzessive aller historischen Gegenstände bemächtigt. Die Digitalisierung löst vom Ende der Geschichte her alle Epochen rückwirkend auf. Der Computer wird uns, das ist Kittlers medientheoretische Wette, nicht nur das Speichermedium für die überlieferte Musik der Vergangenheit sein, sondern er wird seiner Eigenschaft als Supermedium gerecht werden, indem er alle produktiven und rezeptiven Betriebsgeheimnisse einer

20 Rund 180 Seiten des Bands hat Kittler als druckreifes Manuskript hinterlassen (Nachlass im Deutschen Literaturarchiv Marbach). 
beliebigen Musik beliebiger Zeit modelliert. Für den Musikhörer gibt es nichts mehr zu interpretieren, für den Musikhistoriker nichts mehr historisch zu forschen. Man muss sich nur noch in das digitale „absolute Wissen als Endlosschleife“21 einloggen, und schon schaut man nicht mehr mit dem Rankeschen Historikerblick zurück, sondern man „ek-sistiert im“ Musikleben am Hof Karls V., man „ek-sistiert im“ Gesang in der Liturgie eines Klosters der Karolingerzeit, man „ek-sistiert als“ Beethoven, der die Große Fuge komponiert, man „ek-sistiert als“ Odysseus, der zwischen den Sirenenfelsen hindurchsegelt. Die Turingzeit wird die totale Gleichzeitigkeit alles musikalisch Ungleichzeitigen bringen. Kittler sah mediengeschichtlich zwingende Gründe dafür, dass die musikalische Seinsvergessenheit gegenwärtig zu Ende geht. Daher seine eigentümliche Verschränkung von digitaler Posthistoire mit der Hölderlin'schen/Heidegger'schen Rede von der Wiederkehr der Götter, die Kittler desto triftiger scheint, als die Überlegungen von Turing und Heidegger nahezu exakt gleichzeitig formuliert wurden: im Jahr 1936. ${ }^{22}$ Symptomatisch auch, dass Kittler für den vierten Band ursprünglich den Titel „Turinggalaxis und Heideggers Gestell“ vorgesehen hatte ${ }^{23}$ - eine schließlich getilgte Referenz auf Heideggers Technikphilosophie, die allerdings hätte umgedeutet werden müssen: Bei Heidegger war die moderne Technik (das „Ge-stell“) das finale Stadium der totalen Seinsvergessenheit, Kittler begreift sie als das Tor, durch das hindurch alle Ungleichzeitigkeiten überwunden werden und die Götter nahen.

Muss sich die Musikwissenschaft damit beschäftigen? Zum einen wurde darauf schon unter dem Aspekt Totale Mobilmachung weiter oben hingewiesen. Zum anderen: Sie muss es dann, wenn zutrifft, dass die Aussagen der Musikwissenschaft mit einer virtuellen Maschine à la Turing modellierbar sind. Mit dieser Frage sollte es sich die Musikwissenschaft nicht zu leicht machen.

Kittlers zentraler These in Musik und Mathematik kommt man vielleicht am besten mit einer klassischen musikwissenschaftlichen Frage auf die Spur: Wie kommt Musik zu einem Gehalt, zu einer Semantik, zu einer Botschaft? Oder als musikwissenschaftliche Agenda formuliert: Wie kann die Musikwissenschaft einen Gehalt, eine Semantik, eine Botschaft einer bestimmten Musik rekonstruieren? Kittler analysiert in Musik und Mathematik I/1: Aphrodite die singenden Frauen aus Homers Erzählungen. Odysseus befragt die Zauberin und Sängerin Kirke nach dem Weg heim nach Ithaka. Sie gibt ihm eine lange Wegbeschreibung, die sich als eine Mischung aus wahren und falschen Angaben entpuppen wird. Genauer: Die Lügen ergeben sich aus freiem verbalem Weiterspinnen von Wahrheiten; für sich genommen ist Kirkes Wegbeschreibung ein einziges Wortgeplänkel. Kittler behauptet nun, Odysseus hätte sich nie aufgrund des Wortgeplänkels allein in den fremden Gewässern zurechtgefunden. Er hat nur deshalb Vertrauen in die Nennkraft von Kirkes Worten, weil er Kirke als Sängerin kennengelernt hat. Und als sie sang, log sie nicht, konnte sie nicht lügen. Der Gesang ist zwar keine Wahrheitsbedingung der Worte im strikten Sinn, aber doch etwas, was ich oben als Implikatur der Wahrhaftigkeit erläutert habe. Medientheoretisch gesagt: Die Verbalsprache braucht, damit die Worte ihre Wahrheit auch tatsächlich nennen und nicht einfach dahingesagt sind, eine Aura der Wahrhaftigkeit. Die Sirenen im weiteren

21 Kittler, Grammophon Film Typewriter, S. 8.

22 Turing veröffentlichte seine Theorie 1936, Heidegger hielt im selben Jahr den Vortrag „Hölderlin und das Wesen der Dichtung “, in dem Hölderlins Dichterworte von der Wiederkehr der Götter erstmals als seinsgeschichtliche Beschreibung der Gegenwart Verwendung finden; sie finden sich ab diesem Zeitpunkt in zahlreichen seiner Publikationen.

23 So die Verlagsankündigung vom Jahr 2005 vor Erscheinen des ersten Bands; siehe Winthrop-Young, Friedrich Kittler zur Einführung, S. 180. 
Verlauf der Geschichte singen, ohne etwas rein verbal zu sagen, und deshalb singen sie die reinste Wahrhaftigkeit, ohne dass eine Semantik angegeben werden könnte.

Kittlers These zum Gehalt von Musik lautet folglich: „Stimmen bannen, aber sagen nicht wohin. Das [sc. letzteres] nennen wir seit Aristoteles Semantik. “24 Das Bannende der singenden Stimme hat von sich aus keine Semantik, keine Botschaft. Die Semantik des „Sagens wohin“ hingegen bleibt für sich genommen leer und selbstreferenziell. Nennkraft bekommt das Sagen von Worten erst, wenn es in eine Wesensweise der Wahrhaftigkeit gebettet wird. Und Musik ist eine solche Wesensweise, pythagoreisch und vorsokratisch gedacht sogar das Wesen selbst.

Ohne dass der Medienwissenschaftler Kittler in Musik und Mathematik auch nur an einer Stelle noch das Wort „Medium“ in den Mund nimmt, bleibt das Medienapriori weiter gültig. Musik ist das Medium, in das nachfolgende Botschaften mit ihrer Semantik sich einschreiben. Nur wird es bei Homer nicht aus Mutter-, sondern aus Frauenmund empfangen. Das Eingeschriebensein von semantisierter Sprache in Musik bezeichnet Kittler nun mit dem Heidegger'schen Begriff „Lichtung“: „Helden ist im Gegensatz zur Männergier ein Sinn vergönnt, dem Unheil schwant, bevor es eintritt. Wir nennen diesen Sinn die Gegenwart des Geistes, Griechen die von Göttern. Noch haucht und flüstert aus der rechten Hälfte ihres Hirns ein Geheiss zur linken, eine Botschaft, die Gehör und selbst Gehorsam fordert, weil noch kein Gewissen oder Ego heilsame Stimmen in den Wind schlägt. Linke Hirnhälften verstehen blosse Worte, rechte lauschen der Musik. So wesen Göttern an bei ihren Helden, wann immer sie als Vogelfrau Athene oder bärtig junger Hermes aus inneren Ohren in die Lichtung draußen strahlen. Also wenn die Rede scheint wie Licht. Wenn Musik vor Augen tritt." 25

Hier begegnet uns eine weitere maßgebliche Referenz Kittlers, Julian Jaynes' Theorie vom Zusammenbruch des bikameralen Geistes als dem Ausgangspunkt der abendländischen Kultur. ${ }^{26}$ Erst mit Homer und der Schriftkultur, so Jaynes, sei es zu einem permanenten Austausch zwischen der rechten und der linken Hemisphäre des menschlichen Gehirns gekommen. Vorher hätten die imperativische rechte und die ausführende linke Hemisphäre weitgehend getrennt gearbeitet. Die rechte - für Jaynes gleichbedeutend mit der Stimme der Götter - habe der linken eingeflüstert, was zu tun sei. Zusammen mit dem Konzept, dass die rechte Hemisphäre für das emotionale und musische Vermögen der menschlichen Psyche zuständig ist, ergibt sich Kittlers medientheoretische Grundüberlegung: Das Medium Musik (rechte Hemisphäre) wird als ein Lichten, als ein EinRäumen der Möglichkeit des vollen Erfassens und Fürwahrnehmens von Worten (linke Hemisphäre) aufgefasst. Mit der griechischen vokalalphabetischen Schrift, die bei der Verschriftlichung der homerischen Gesänge historisch erstmals auftaucht, kommt es zu einer fugenlosen Interaktion beider Hemisphären. In der vokalalphabetischen Schrift sind die bedeutungstragenden und die klanglichen Elemente in ein und dasselbe Medium von 24 Buchstaben eingegangen. Fortan werden beim Sprechen, Schreiben und Singen das Erfassen von Bedeutungen (linke Hemisphäre) und der Appell von Wahrheit und Dringlichkeit der Bedeutungen (rechte Hemisphäre) im selben Medium prozessiert. Mit diesem Zusammenbruch des Zwei-Kammern-Bewusstseins ist für Kittler der Keim der Seinsvergessenheit gesetzt. Bewusst sind uns nämlich nur die Gehalte der vormals linken Hemisphäre. Die

24 Musik und Mathematik I/1: Aphrodite, S. 26.

25 Ebd., S. 28.

26 Julian Jaynes, The Origin of Consciousness in the Breakdown of the Bicameral Mind, Boston/MA 1976, dt.: Der Ursprung des Bewußtseins durch den Zusammenbruch der bikameralen Psyche, Reinbek 1988. Auch diese Referenz legt Kittler nur selten offen, hier in: Musik und Mathematik I/1: Aphrodite, S. 28. 
psychischen Funktionen der Beglaubigung und des Dringlichkeitsappells der vormals rechten sind als solche keine Gegenstände des Bewusstseins, ohne dass sie aufgehört hätten, im Verborgenen weiterzuarbeiten.

Wie oben beim Thema Militär muss man Jaynes' strittige Theorie keineswegs in allen Details akzeptieren, um die Behauptung, Musik sei ein Lichten, ein Raum-geben, ein appellierendes Beglaubigen von Semantiken, nicht aber ein Trägermedium von Semantiken oder gar selbst semantisch, zur Prüfung zuzulassen. Die Wette, dass es sich mit dem menschlichen Bewusstsein so verhält, wird nicht dadurch entschieden, dass neurologisch dies oder jenes der Fall ist, sondern dadurch, dass es eine denkerische Notwendigkeit für ein Ineinander von begrifflichem Gehalt und Beglaubigungsinstanzen für Gegenstände des menschlichen Bewusstseins gibt oder nicht. Auf vielen disziplinären Wegen wird diese Behauptung gegenwärtig überprüft. Nehmen wir an, sie stimmt, wofür es viele Indizien gibt. Dann ergeben sich für die Musikwissenschaft folgende Perspektiven:

1. Kittler macht die Behauptung historisch in der Gründungsphase der abendländischen Kultur fest. Seine Behauptung beinhaltet also, dass sie die gesamte abendländische Musik prägt, wenngleich möglicherweise in den beschriebenen Verfallsstadien. Daraus ergibt sich ein gewaltiges interdisziplinäres Forschungsprogramm zwischen der Musikwissenschaft und derjenigen Disziplin, aus deren Gegenstandsbereich jeweils die Semantik stammt. Allerdings erzwingt die Behauptung eine disziplinäre Gewichtung, die in der gegenwärtigen interdisziplinären Forschungslandschaft kaum anzutreffen ist: Die Musikwissenschaft müsste eine Art Grundlagendisziplin für die jeweils andere Disziplin sein. Die Musikwissenschaft hätte die gründenden und verifikativen Prozesse von Semantiken der je anderen Disziplin zu explizieren.

2. McLuhans erstem medientheoretischen Hauptsatz „The medium is the message“ will Kittler im alten Griechenland zur Evidenz verhelfen. Im Vergleich mit Kittlers früheren medienwissenschaftlichen Arbeiten wird mit dieser Beschreibung der Wirksamkeit von Medien ein neuer Akzent gesetzt. In vielen älteren medientheoretisch inspirierten Studien nicht nur Kittlers, sondern auch von in die Kulturwissenschaften abgesunkenen Adaptionen konnte man den Eindruck gewinnen, im weißen Rauschen des Medienaprioris verschwinde jeglicher Gehalt und alle wahrheitswertfähige Bedeutung. Das dürfte einer der unausgesprochenen Gründe gewesen sein, warum die etablierte Musikwissenschaft vor medientheoretischen Ansätzen zurückschreckte. Heideggers Begriff der Lichtung mag spekulativ und metaphorisch sein, aber er stellt McLuhans so berühmten wie unpräzisen und missverständlichen Hauptsatz klar:

- Ein Medium bringt von sich aus keine wahrheitswertfähige Semantik mit (die Stimmen sagen nicht wohin).

- Ein Medium vertilgt wahrheitswertfähige Semantiken aber auch nicht (Odysseus findet mit, nicht entgegen Kirkes Beschreibung den Weg).

- Ein Medium verhilft einer wahrheitswertfähigen Semantik zu ihrer Faktizität (Stimmen bannen).

3. Kittlers Ansatz wurzelt in seiner Grundhaltung erstens in Heideggers spätem Denken von Ereignis und Sprache, zweitens in Lacans und Jaynes' kulturpsychologischen Theorien, drittens in Foucaults Diskurstheorie. ${ }^{27}$ Bemerkenswert ist, dass er gegenwärtig auch un-

27 Neben Michel Foucault, Les mots et les choses, Paris 1966 (dt. Die Ordnung der Dinge, Frankfurt a. M. ${ }^{15} 1999$ ) bezieht sich Kittler häufig auf Foucault, Histoire de la sexualité, Paris 1976-1984. An letzterer Referenz ließe sich Kittlers beständiges Überblenden von Musik und Liebe explizieren, ein Zusammenhang, den ich im vorliegenden Beitrag ausspare. Die französischen Phonozentristen Derrida (La voix et 
abhängig von dieser Tradition an Aktualität gewinnt. So nähert sich ihr Albrecht Wellmer von Adornos Musikphilosophie her an, ${ }^{28}$ von Noam Chomskys Universalgrammatik aus versuchen es Jonah Katz und David Pesetsky, ${ }^{29}$ Andreas Luckner von der analytischen Sprachphilosophie, ${ }^{30}$ ich selbst versuche Ansätze von Alain Badiou, Jean-Luc Nancy und Herbert Paul Grice weiterzudenken. ${ }^{31}$

Muss sich die Musikwissenschaft dafür interessieren? Ja, sie sollte, denn hier wird für die alte Frage, wie Musik zu einem Gehalt kommt und welche Verbindung Wortsemantiken und ihre Musikalisierung eingehen, eine diskutable These formuliert. Die oft an der Oberfläche bleibenden musikwissenschaftlichen Deutungen in den wortgebundenen musikalischen Gattungen von der Wortsemantik her (Musik vertont den Text, Musik deutet den Text aus, Musik repräsentiert den Text, Musik geht am Text entlang usw.) werden mit ihr vom Kopf auf die Füße gestellt. Der so einfache wie grundstürzende Umstand, dass Sprachbedeutungen oder auch Sinnzusammenhänge etwa in Politik oder Religion ihren eigenen Anspruch auf Nennkraft nicht von sich aus einlösen können und dafür einer Verifizierung bedürfen, und dass Musik (im weitesten Sinne, nämlich auf den beiden oben beschriebenen Ebenen) offenkundig diese Verifikation zu leisten imstande ist, bringt die Musik in ein Grund-legendes Verhältnis zur Sprache. Entsprechend groß sind Last und Verantwortung der Musikforschung, die stiftende Rolle der Musik für die Existenzweisen Religion, Politik oder auch Liebe zu erläutern.

\section{Mathematik}

Warum Musik und Mathematik? Die fortschreitende Seinsvergessenheit, die Kittler in seinem unvollendeten Projekt darstellen wollte, ist auch eine Verfallsgeschichte des und. Um das nachzuvollziehen, müssen wir das Medium Sprache hinzuziehen, und zwar in der eben diskutierten Hinsicht, dass Sprache in einem vorsprachlichen Medium gegründet werden muss, um eine Sache wirklich zu benennen. Und dieses Medium, so Kittler, ist bei den alten Griechen Musik - und Mathematik mit ihr im Verbund.

Was es meint, dass Sprache eine Sache wirklich benennt, dafür verweist Kittler gerne auf einen weiteren Freiburger Gewährsmann, bei dem er im Unterschied zu Heidegger noch selbst studiert hatte, den Altphilologen Johannes Lohmann. Eine Grundreferenz Kittlers ist folgende These Lohmanns: Ein Wort werde bei den vorsokratischen Griechen nicht als willkürliches Zeichen aufgefasst, dem man arbiträre Bedeutungen zuordnen könne, „son-

le phenomène, Paris 1967, dt. Die Stimme und das Phänomen, Frankfurt a. M. 2003) und Roland Barthes (Die Körnung der Stimme. Interviews 1962-1980, Frankfurt a. M. 2002) spielen hingegen nur beim frühen Kittler eine Rolle. In seinen letzten Jahren hat sich Kittler namentlich von Derrida polemisch abgewandt.

28 Albrecht Wellmer, Versuch über Musik und Sprache, München 2009.

29 Jonah Katz und David Pesetsky, The Identity Thesis for Language and Music (Januar 2011), <http://ling. auf/net/lingBuzz/000959>, 4.1.2012.

30 Andreas Luckner, „Musik - Sprache - Rhythmus. Bemerkungen zu Grundfragen der Musikphilosophie“, in: Musikphilosophie, hrsg. von Ulrich Tadday (= Musik-Konzepte, Sonderband), München 2007, S. 34-49.

31 Rainer Bayreuther, Was ist religiöse Musik?, Badenweiler 2010, bes. Kap. 43-50; ders., Der musische Staat (in Vorb.). 
dern das Wort ist der Name einer Sache: als Name, onoma, hat das Wort eine Kraft, dynamis, die Kraft zu nennen." 32

Kittler behauptet nun, diese Kraft hat nicht nur das gesprochene Wort. Die Kraft hat auch das griechische Vokalalphabet, und zwar deshalb, weil es im Unterschied etwa zur jüdischen Konsonantenschrift, die im Prinzip eine Silbenschrift ist, oder zu den ägyptischen Hieroglyphenschriften, die eine Kombination von Ganzwortzeichen und Einzelkonsonantenzeichen sind, die Phoneme des ausgesprochenen Worts aufzeichnet. Eine Vokalschrift bildet die aufeinanderfolgenden Mikromomente des Sprechens selber ab. Während die Konsonanten- und Hieroglyphenschriften aufgrund ihrer Nachrangigkeit von vornherein auf Verifikationen ihrer Wahrhaftigkeit verzichten und diese der ausgesprochenen Sprache überlassen, erheischt bei den Griechen das geschriebene Wort dieselbe Verifikation wie das gesprochene. In der gesprochenen Sprache wird diese Verifikation vom einhüllenden Sprachklang geleistet, vom - wenn man so will - gesungenen Wort. Dazu sucht Kittler ein Äquivalent für die Schrift. Dieses besteht im Wesentlichen aus drei Merkmalen: 1. Die griechische Schrift zeichnet auch die Vokale auf. Das heißt, sie funktioniert nicht mehr symbolisch, sondern zeichnet ihre eigene Klanglichkeit auf. 2. Nach einer kurzen historischen Spanne schreiben die Griechen mit ihren 24 Alphabetzeichen auch die Töne der drei Tongeschlechter und die Zahlen auf. Das ist ein Indiz dafür, dass sich die Griechen das Verikfikationsmedium Klang nicht als einen bloßen psychischen Effekt des Schmeichelns, Überredens, Vertrautseins, mütterlichen Geborgenseins etc. vorstellten, sondern dass dieses Medium eine wesentliche Struktur der Welt ist: die Zahlen und ihre elementaren Verhältnisklassen, und die Tongeschlechter, die aus den Verhältnisklassen der Zahlen hervorgehen. Kurz: Die vokalalphabetisch geschriebene Sprache oder das in Tonbuchstaben angeschriebene Musikstück enthalten in nuce ihre Verifikationsmedien in sich selbst.

Von hier aus kann die Seinsverlassenheit, die Kittler mit dem allmählichen Verlust des und verbindet, klarer gesehen werden. Bei Platon und Aristoteles sind Sprachphilosophie, Musikwissenschaft und Arithmetik eigenständige Disziplinen geworden. Der materialistische Verifikationszusammenhang ist zerbrochen, ihre Beglaubigung erhalten Worte, Zahlen und Klänge nur über das geistige Konstrukt der Ideen. Die Ausdifferenzierung der Künste, etwa in der literarischen Gattung der Tragödie oder im allmählichen Missverstehen der alten Tongeschlechter beim freien Setzen von Tönen, ist ein epochales Vergessen der Tatsache, dass sich Wort-Sätze und Ton-Sätze in ein welthaltiges Medium - Klang oder Zahl oder beides - einschreiben müssen, damit der literarische oder musikalische Akteur über das Stück Literatur oder Musik auch tatsächlich in ein Weltverhältnis kommt.

Kittlers wilde Deutung der Odyssee hat die Gräzisten und die Historiker auf den Plan gerufen. Erstere reiben sich an den eigenwilligen und in vielen Details falschen HomerÜbersetzungen im ersten Teilband von Musik und Mathematik I. Letztere heben die großen Unsicherheiten von Kittlers - von dem Altphilologen Barry Powell übernommenen ${ }^{33}$ Hypothese hervor, das Vokalalphabet sei eigens zur Aufzeichnung von Homers Epen erfunden worden. Sind diese Einwände für die Musikwissenschaft ein Grund, seine Überlegungen pauschal zu verwerfen? Nein. Die für die Musikforschung relevante Überlegung von Musik und Mathematik lautet: Die übliche Frage nach dem Gehalt eines Musikstücks

32 Johannes Lohmann, „Musiké und Logos“, in: ders.: Musiké und Logos. Aufsätze zur griechischen Philosophie und Musiktheorie, hrsg. von Anastasios Giannarás, Stuttgart 1970, S. 1-15, hier: S. 14. Auch Lohmanns Aufsatz „Die griechische Musik als mathematische Form“ (ebd., S. 17-25) hat für Kittler große Bedeutung.

33 Barry B. Powell, Homer and the Origin of the Greek Alphabet, Cambridge/MA 1991. 
ist falsch gestellt; die sinnvolle Frage ist, welche Rolle ein Musikstück bei der Genese und Beglaubigung anderweitiger Gehalte spielt, die im Reifestadium der Auskonkretisierung nicht-mehr-musikalische Gehalte sind. Diese Fragerichtung ist falsifizierbar. Und die Antwort darauf, ob die Fragerichtung richtig oder falsch ist, hängt weder davon ab, ob das Vokalalphabet tatsächlich von Homer und seinem Sekretär auf der Insel Euböa 800 v. Chr. erfunden wurde, noch davon, dass Kittlers Homerinterpretation in allen Details philologisch fehlerfrei ist. Ob Kittlers Fragerichtung richtig ist, muss musikästhetisch und musikphilosophisch entschieden und durch musikalische Analyse belegt werden. Von der Antwort darauf hängt nichts Geringeres ab als dies: Kann die Musikwissenschaft eine grundlegende Deutungskompetenz über die Grundweisen der menschlichen Existenz - Religion, Politik, Liebe - beanspruchen? 